\title{
Multisoliton excitations in long Josephson junctions
}

Dueholm, B.; Levring, O. A.; Mygind, Jesper; Pedersen, Niels Falsig; Sørensen, O. H.; Cirillo, M.

Published in:

Physical Review Letters

Link to article, DOI:

10.1103/PhysRevLett.46.1299

Publication date:

1981

Document Version

Publisher's PDF, also known as Version of record

Link back to DTU Orbit

Citation (APA):

Dueholm, B., Levring, O. A., Mygind, J., Pedersen, N. F., Sørensen, O. H., \& Cirillo, M. (1981). Multisoliton excitations in long Josephson junctions. Physical Review Letters, 46(19), 1299-1302.

https://doi.org/10.1103/PhysRevLett.46.1299

\section{General rights}

Copyright and moral rights for the publications made accessible in the public portal are retained by the authors and/or other copyright owners and it is a condition of accessing publications that users recognise and abide by the legal requirements associated with these rights.

- Users may download and print one copy of any publication from the public portal for the purpose of private study or research.

- You may not further distribute the material or use it for any profit-making activity or commercial gain

- You may freely distribute the URL identifying the publication in the public portal

If you believe that this document breaches copyright please contact us providing details, and we will remove access to the work immediately and investigate your claim 
quency, 0.76 MG, has been identified. The apparent effective mass and Dingle temperature values are 1.1 and $3 \mathrm{~K}$, respectively. The oscillations appear only for $H$ approximately parallel to the $c$ axis, requiring the presence of closed orbits in the $a-b$ plane. Their frequency varies as $(\cos \theta)^{-1}$, implying that the orbits are open along the $c$ axis, consistent with the magnetoresistance. Therefore the Fermi surface appears to be essentially two dimensional. The data also imply that the material is a compensated metal, with both electron and hole surfaces. Anomalies have been found associated with the effect, which may be related to many-body correlations and to magnetic breakdown.

We wish to thank D. L. Overmyer for extensive technical aid. This work supported by the U. S. Department of Energy under Contract No. DEAC04-76-DP00789 at Sandia National Laboratories (a U. S. Department of Energy facility) and at IBM by the U. S. Office of Naval Research.

${ }^{1}$ K. Bechgaard, C. S. Jacobsen, K. Mortensen, H. J. Pedersen, and N. Thorup, Solid State Commun. $\underline{33}$, 1119 (1980).

${ }^{2}$ D. Jerome, A. Mazaud, M. Ribault, and K. Bechgaard, J. Phys. (Paris), Lett. 11 , L95 (1980).
${ }^{3}$ K. Andres, F. Wudl, D。B. McWhan, G. A. Thomas, D. Nalewajek, and A. L. Stevens, Phys. Rev. Lett。 45, 1449 (1980).

${ }^{4}$ R. L. Greene and E. M. Engler, Phys. Rev. Lett. 45, 1587 (1980).

${ }^{5}$ W. M. Walsh, Jr., F. Wudl, G. A. Thomas, D. Nalewajek, J. J. Hauser, P. A. Lee, and T. Poehler, Phys. Rev. Lett. 45, 829 (1980).

${ }^{6}$ P. M. Chaikin, G. Gruner, E. M. Engler, and R. L. Greene, Phys. Rev. Lett. 45, 1874 (1980).

${ }^{7}$ K. Mortensen, Y. Tomkiewicz, T. D. Schulz, and E. M. Engler, to be published.

${ }^{8} \mathrm{See}$, for example, various articles in Chemistry and Physics of One-Dimensional Metals, edited by

H. J. Keller (Plenum, New York, 1977).

${ }^{9}$ J. E. Schirber, Cryogenics 10, 418 (1970).

${ }^{10}$ J. K. Kwak, J. E. Schirber, R。 L。 Greene, and E. M. Engler, to be published.

${ }^{11}$ C. S. Jacobsen, K. Mortensen, M. Weger, and

K. Bechgaard, to be published.

${ }^{12}$ E. Fawcett, Adv. Phys. 13, 139 (1964).

${ }^{13}$ C. S. Jacobsen, K. Mortensen, N. Thorup, D. B. Tanner, M. Weger, and K. Bechgaard, to be published.

${ }^{14}$ L. M. Roth and P. N. Argyres, in Semiconductors and Semimetals, edited by R. K. Willardson and A. C. Beer (Academic, New York, 1966), Vol. I, p. 159.

${ }^{15} \mathrm{To}$ find the field dependence of the $150-\mathrm{Hz}$ signal one must include on the right-hand side of Eq. (2) a Bessel function factor $J_{3}(Z)$, where $Z=2 \pi r F H_{\bmod } / H^{2}$.

${ }^{16} \mathrm{P}$. Schlottman and L。 M. Falicov, Phys. Rev. Lett. 38, 855 (1977)。

${ }^{17}$ H. J. Pedersen, J. C. Scott, and K. Bechgaard, Solid State Commun. 35, 207 (1980).

\title{
Multisoliton Excitations in Long Josephson Junctions
}

B. Dueholm, O. A. Levring, J. Mygind, N. F. Pedersen, and O. H. Soerensen Physics Laboratory I, The Technical University of Denmark, DK-2800 Lyngby, Denmark

and

M. Cirillo

Istituto di Fisica, Universita di Salerno, Salerno, Italy, and Physics Laboratory I, The Technical University of Denmark, DK-2800 Lyngby, Denmark (Received 23 December 1980)

\begin{abstract}
The microwave emission from long Josephson tunnel junctions dc-current biased on zero-field and Fiske steps has been measured. The frequency and power variation on all steps of the narrow-linewidth radiation near the fundamental cavity-mode frequency and the observed transitions between different modes on a given step may be understood in a picture of multifluxon excitations with propagation of different bunched fluxon configurations depending on the current and magnetic field bias.
\end{abstract}

PACS numbers: $74.50 .+\mathrm{r}, 73.40 . \mathrm{Rw}, 84.40 . \mathrm{Cb}$

The concept of soliton excitations of Josephson tunnel junctions was introduced in order to explain the so-called zero-field steps (ZFS) in the current-voltage characteristic of long tunnel junctions. ${ }^{1}$ The soliton or fluxon is a $\pm 2 \pi \mathrm{kink}$ in the phase difference across the barrier encom- 
passing one quantum of magnetic flux. The width is of order of the Josephson penetration depth $\lambda_{\mathrm{J}}$. The fluxon propagates with a velocity $u$ governed by a perturbed sine-Gordon equation ${ }^{2}$ in a periodic motion with repeated reflections at the open ends of the junction. Each reflection creates a voltage pulse. A single fluxon on the Josephson line of length $L$ hence produces a periodic pulse train of frequency $f_{1}=u / 2 L$ which may be detected at either end of the junction. On the $N$ th ZFS the steady state involves an $N$-fluxon excitation of the junction and $N$ pulses are produced in each period $1 / f_{1}$. Hence, the frequency spectrum of the voltage monitored at one end of the junction depends on the distribution in time of the $N$ pulses within the period.

In recent computer simulations of the two-fluxon excitation on a finite line, two steady-state configurations were observed. ${ }^{3}$ Either a fluxon-antifluxon pair moved as expected ${ }^{1}$ in a perfectly inflection-symmetric mode with respect to the midpoint of the line or a two-fluxon "bunch" was formed moving as one entity. The corresponding fundamental frequency of the voltage waveform at the end of the junction was $2 f_{1}$ or $f_{1}$ in the two cases, respectively. Stable multifluxon bunches have been observed also on mechanical analog models of the finite Josephson line. ${ }^{4,5}$ On the infinite line the multifluxon bunches are stable if the sine-Gordon model is augmented by additional surface damping terms. ${ }^{6}$

In this Letter we report on detailed measurements of $10-\mathrm{GHz}$ radiation from long Josephson tunnel junctions biased on the ZFS or on the Fiske steps which appear when an external magnetic field is applied. ${ }^{7}$ In contrast to earlier observations ${ }^{8}$ and predictions based on the fluxon-antifluxon array model $^{9}$ we observed radiation at 10 GHz on all steps. This is consistent with an interpretation assuming stable periodic motion of bunched multifluxons.

The samples were $\mathrm{Nb}-\mathrm{Nb}$-oxide- $\mathrm{Pb}$ tunnel junctions in an overlap geometry which favors a uniform dc-current bias distribution along the length of the junction. ${ }^{10}$ The $\mathrm{Nb}$ films were deposited by rf sputtering and thermally oxidized. The $\mathrm{Pb}$ counter electrodes were deposited in a subsequent thermal evaporation. Junction and film geometries were defined by photolithographic masks and chemical etching. ${ }^{11}$ Six samples covering the range $2 \lesssim L / \lambda_{\mathrm{J}} \lesssim 22$ were tested and the results presented below are typical for all samples. The emitted microwave radiation was detected with a sensitive superheterodyne receiver coupled via a 50- $\Omega$ microstrip transmission line and a narrow capacitive gap to one end of the junction.

In a short sample $\left(L \simeq 0.5 \mathrm{~mm}, L / \lambda_{\mathrm{J}} \simeq 2\right)$ we observed three ZFS with a voltage spacing of 42 $\mu \mathrm{V}$. On all three steps radiation at $f_{1}=10 \mathrm{GHz}$ was observed. This is in contrast to earlier model calculations ${ }^{9}$ which predicted radiation with a fundamental at half the Josephson frequency $\left(f_{\mathrm{J}}=2 e V_{\mathrm{dc}} / h\right)$, i.e., at 10,20 , and $30 \mathrm{GHz}$ on the steps at 42,84 , and $126 \mu \mathrm{V}$, respectively. The frequency shift $\Delta f$ versus the voltage increments $\Delta V_{\mathrm{dc}}$ along the steps are plotted in Fig. 1. The points follow a linear dependence according to a modified Josephson voltage-frequency relation $\Delta f / \Delta V_{\mathrm{dc}}=2 e / n h$, where the index $n=2,4,6$ $\left(n=f_{\mathrm{J}} / f_{1}\right)$ for the first, second, and third ZFS, respectively. The emitted linewidth was consistently less than $7 \mathrm{kHz}$ which was the frequency resolution limit of the receiver. ${ }^{12}$

In Fig. 2(a) the detected $10-\mathrm{GHz}$ power is shown versus dc bias current on the second ZFS $(n=4)$ of the same junction as in Fig. 1. Very little power is observed on the low-current part of the step. This behavior is distinctly different from the current dependence of the second harmonic $\left(2 f_{1}\right)$ as demonstrated in Fig. 2(b). This result is obtained from a long junction $\left(L \simeq 1 \mathrm{~mm}, L / \lambda_{\mathrm{J}}\right.$ $\simeq 3$ ) where the fundamental is at $f_{1}=5 \mathrm{GHz}$. A mode transition is clearly evident in the lowcurrent part of the step. As the bias current is increased a sharp peak in the emitted $10-\mathrm{GHz}$ power is observed followed by low-level radiation almost independent of bias. The transition is accompanied by a discontinuous jump in fre-

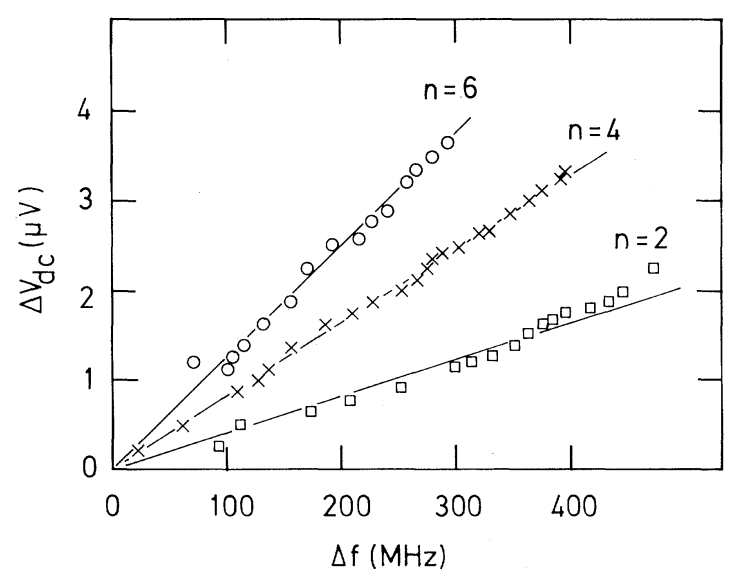

FIG. 1. Frequency shift vs voltage increment on zero-field steps $n=2,4$, and 6 . The lines are drawn with slope $\Delta f / \Delta V_{\mathrm{dc}}=484 / n(\mathrm{MHz} / \mu \mathrm{V})$. 


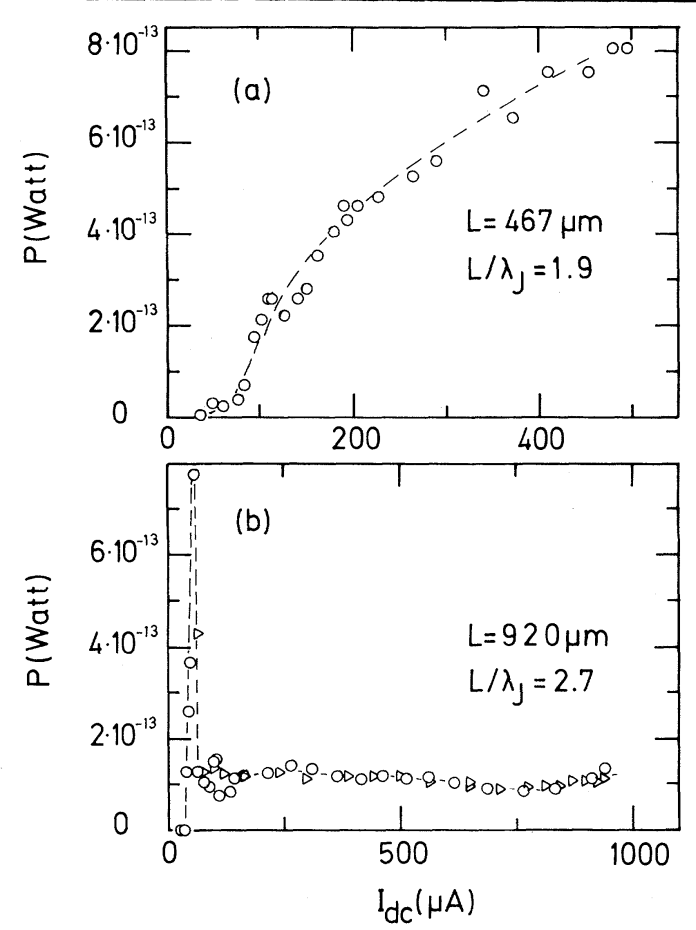

FIG. 2. Measured microwave power at $10 \mathrm{GHz}$ from the second ZFS $(n=4)$. (a) $\frac{1}{2}-\mathrm{mm}$ junction, $L / \lambda_{\mathrm{J}} \simeq 2$. (b) $1-\mathrm{mm}$ junction, $L / \lambda_{\mathrm{J}} \simeq 3$. Circles: increasing bias; triangles: decreasing bias.

quency of about $50 \mathrm{MHz}$. The results presented in Fig. 2 are consistent with the numerical results of Erne and Parmentier. ${ }^{3}$ The narrow peak in Fig. 2(b) is first harmonic radiation generated by the inflection-symmetric fluxon-antifluxon pair whereas the low-level radiation is the second harmonic of the $5-\mathrm{GHz}$ radiation associated with the two-fluxon bunch. In Fig. 2(a) only first harmonic radiation from the two-fluxon bunch can be seen because the inflection-symmetric mode which may be excited in the low-current part of the step radiates at $20 \mathrm{GHz}$ and higher harmonics.

In all junctions about $L / \lambda_{\mathrm{J}}$ ZFS were observed of gradually decreasing magnitude. ${ }^{13}$ The highorder steps $(n>6)$ exhibited fine structure in the form of branching and small hysteresis loops. The observed radiation spectra suggest that the different branches on a given ZFS correspond to different fluxon configurations and that radiation at $10 \mathrm{GHz}$ is generated on all ZFS. This is illustrated in Fig. 3(a) showing the fifth ZFS $(n=10)$ of a long junction ( $L \simeq 1 \mathrm{~mm}, L / \lambda_{\mathrm{J}} \simeq 6, f_{1}=5 \mathrm{GHz}$ ). As the bias point is swept from $A$ to $B$ no $10-\mathrm{GHz}$ radiation is detected. At $B$ the voltage jumps to

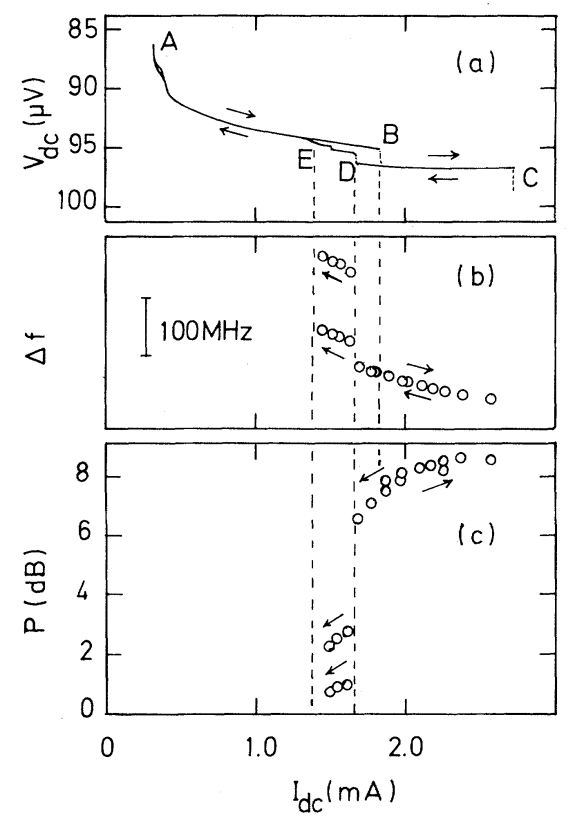

FIG. 3. Mode transitions in a 1- $\mathrm{mm}$ junction biased on the fifth ZFS $(n=10)\left(L=1 \mathrm{~mm}, L / \lambda_{\mathrm{J}} \simeq 6\right)$. (a) $\mathrm{dc}$ voltage vs current. (b) Frequency shift vs current. (c) Microwave power at $10 \mathrm{GHz}$ vs current.

another branch and a signal appears. The frequency and the power of the observed signals are plotted versus bias current in Figs. 3(b) and 3 (c), respectively. Both frequency and power vary smoothly as the bias point increases from $B$ to $C$. For decreasing bias, however, another discontinuous transition takes place at $D$ and a branch is reached where signals appear simultaneously at two frequencies with a separation of $\sim 100 \mathrm{MHz}$. At point $E$ both signals disappear again as the voltage completes the hysteresis loop and returns to branch $A B$. All signals satisfy $\Delta f / \Delta V_{\mathrm{dc}}=2 \times(2 e / 10 h)$ consistent with a second harmonic signal, if a simple average is used for $\Delta f$ on the branch with two signals.

We suggest that the complex behavior depicted in Fig. 3 can be interpreted in terms of fluxons. A total of five fluxons take part and in order to explain emission of $10-\mathrm{GHz}$ radiation we must assume that the fluxons move in a 5 bunch, a $(4+1)$ bunch, or in a $(3+2)$ bunch rather than as five individual fluxons uniformly distributed along the line. The latter mode produces $25-\mathrm{GHz}$ radiation whereas the bunched modes all produce the fundamental $\sim 5-\mathrm{GHz}$ radiation provided all bunches move with the same velocity. It is conceivable that the velocities of the different configurations depend on the number of fluxons involved 
and hence might produce two slightly different frequencies. The two signals observed simultaneously may, however, also be alternating and hence represent an unstable regime where the junction jumps between two different states. The absence of observable radiation on branch $A B$ may reflect excitation of the uniform mode with a fundamental at $25 \mathrm{GHz}$.

The frequency of the radiation from ZFS may be tuned over several hundred $\mathrm{MHz}$ if a dc magnetic field is applied. ${ }^{14}$ This is not surprising since the magnetic field changes the boundary conditions. More noticeable is the appearance of Fiske steps (FS) at voltages $V_{\mathrm{dc}}=n h f_{1} / 2 e, n$ $=1,2,3, \ldots$, which coincide with the ZFS for even $n$. In short junctions $\left(L / \lambda_{\mathrm{J}} \ll 1\right)$ the FS are interpreted as the dc manifestation of junction cavity modes excited by the ac-Josephson effect. ${ }^{15}$ Preliminary measurements of the $10-\mathrm{GHz}$ radiation emitted from our long junctions biased on FS show exactly the same features as the radiation from ZFS. The fundamental frequency, $f_{1}$, is excited on all steps. The frequency versus voltage shift $\Delta f / \Delta V_{\mathrm{dc}}=2 e / n h$ for every $n$ 。 Mode transitions (branching) are seen on FS of high order. The linewidth of the radiation is narrow and determined by external noise in the dc current and magnetic field bias. We are led to the conclusion that also the FS in long junctions are due to propagating fluxons.

In order to reproduce the voltage position of the FS within the fluxon picture multiples of a half-integer number of fluxons must on the average be present on the Josephson line. The first FS $(n=1)$ may, e.g., be generated by a mode suggested by Samuelsen ${ }^{16}$ in which a fluxon propagates in the direction of energy flow defined by the external magnetic field. At the end of the junction the fluxon is annihilated and part of its energy is released and reflected in the form of plasmons or breather oscillations which in turn launches a new fluxon from the opposite end. All the odd-order FS may be explained by similar processes where $n$ fluxons propagate in one direction and only $n-1$ are reflected.

In conclusion, we state that all our observations of microwave emission from long Josephson tunnel junctions are reconciled with the fluxon picture if the stable steady states are the collective motion of multifluxon bunches on major parts of the zero-field and Fiske steps. In particular, the narrow linewidth of the emitted radiation (limited by bias noise) strongly supports this conclusion. Finally, very recent computer experiments ${ }^{17}$ based on the sine-Gordon model with parameters relevant to our experiments have confirmed the interpretation in terms of bunched-fluxon propagation.

The samples were fabricated at the University of Salerno, Italy, and we want to acknowledge the fruitful cooperation with the Josephson effect group there. Also, many stimulating discussions with A. C. Scott and M. R. Samuelsen are gratefully acknowledged. The project was supported in part by the Danish Natural Science Research Council and by the Danish-Italian Cultural exchange program.

${ }^{1}$ T. A. Fulton and R. C. Dynes, Solid State Commun. 12,57 (1973).

${ }^{2}$ A. C. Scott, F. Y. F. Chu, and S. A. Reible, J. Appl. Phys. 47, 3272 (1976).

${ }^{3}$ S. N. Erné and R. D. Parmentier, in Proceedings of the Applied Superconductivity Conference, Santa Fe, 1980 (to be published).

${ }^{4} \mathrm{~K}$. Nakajima, T. Yamashita, and Y. Onodera, J. Appl. Phys. 45, 3141 (1974).

${ }^{5}$ M. Cirillo, M.Sc. thesis, University of Salerno, 1979 (unpublished); M. Cirillo, R. D. Parmentier, and B. Savo, to be published.

${ }^{6} \mathrm{~W}$. J. Johnson, Ph.D. thesis, University of Wisconsin, 1968 (unpublished).

${ }^{7}$ D. D. Coon and M. D. Fiske, Phys. Rev. 138, A744 (1965).

${ }^{8}$ T. A. Fulton and L. N. Dunkleberger, Rev. Phys. Appl. 9, 299 (1974).

${ }^{9}$ G. Costabile, R. D. Parmentier, and B. Savo, in Proceedings of the Fifteenth International Congress of Refrigeration, Venizia, September 1979 (unpublished), Paper No. A 1/2-5.

${ }^{10} \mathrm{~W}$. J. Johnson and A. Barone, J. Appl. Phys. $\underline{41}$, 2958 (1970).

${ }^{11}$ V. Lacquaniti, G. Marullo, and R. Vaglio, IEEE Trans. Magn. 15, 593 (1979).

${ }^{12}$ This narrow linewidth is interesting in view of an application as a radiation source.

${ }^{13}$ In some cases a large number of small irregular steps were observed with the same spacing as the ZFS. We believe these steps to be of a different nature than the ZFS.

${ }^{14}$ T. F. Finnegan, J. Toots, and J. Wilson, in Low Temperature Physics, LT-14, edited by M. Krusius and M. Vuorio (American Elsevier, New York, 1975), Vol. 4, p. 184 .

${ }^{15}$ I. O. Kulik, Zh. Tekh. Fiz. 36, 155 (1969) [Sov. Phys. Tech. Phys. 12, 111 (1967)].

${ }^{16} \mathrm{M}$. R. Samuelsen, private communication.

${ }^{17}$ P. L. Christiansen, P. S. Lomdahl, A. C. Scott, O. H. Soerensen, and J. C. Eilbeck, to be published. 